Imaging Lymph Nodes with Nanoparticles DOI: 10.1371/journal.pmed.0010067

Accurate staging of cancers is one of the most important parts of the work up of patients for both prediction of prognosis and determination of the most appropriate treatment. And an essential part of this work up is assessing whether or not there has been lymphatic spread. Current methods include surgical removal of nodes for examination and various types of imaging, ranging from ultrasound to newer technologies such as magnetic resonance imaging (MRI). All these methods have problems; some are very invasive, others are very time consuming, and none are completely reliable.

However in one of the more exciting crossovers from chemistry into medicine, researchers have developed nanoparticles to improve the diagnostic accuracy of MRI. The nanoparticles contain a central superparamagnetic iron oxide core and are covered by dextran, imparting long circulation times and biocompatibility. When injected intravenously, the nanoparticles localize to lymphoid tissue, and are internalized into macrophages. There is then a decrease in signal intensity on T2- and T2*-weighted images, and when metastases are present there is a recognizably abnormal pattern on MRI scans.

In a previous paper published in the New England Journal of Medicine, Ralph Weissleder and colleagues described using these nanoparticles to assess lymphoid spread in patients with prostate cancer. Now, in a paper published in this month's PLoS Medicine, they have gone further by extending the analysis to patients with different types of cancer, and producing an algorithm that allows semiautomation of the procedure.

The authors developed the algorithm in a training group of 36 patients and then validated it in a group of 34 patients. The results are encouraging: the analysis showed a sensitivity of $98 \%$ (95\% confidence interval, $88 \%-99 \%$ ) and a specificity of $92 \%$ (95\% confidence interval, $87 \%-$ $96 \%$ ). The advantages of automating this procedure are substantial, not least because it can remove the problem of different observers assessing data differently.

And what is more, once the data have been collected and assessed it is possible to reconstruct a virtual picture of the patient's lymph nodes, thus potentially allowing accurate surgical removal of the nodes.

Harisinghani MG, Weissleder R (2004) Sensitive, noninvasive detection of lymph node metastases. DOI: 10.1371 /journal. pmed.0010066

\section{Sleep Duration}

\section{Affects Appetite-Regulating Hormones}

DOI: 10.1371/journal.pmed.0010068

Some of us, when awake in the middle of the night, feel an urge to visit the kitchen. This could explain results of previous studies that have shown a link between short sleep duration and high body mass index (BMI). But a study by Emmanuel Mignot and colleagues suggests that it's not just the additional snacking opportunities that make short sleepers more likely to be overweight.

Intrigued by the connection between sleep and BMI, and by recent studies showing that sleep deprivation in laboratory settings can cause a decrease in serum levels of leptin, a hormone known to control appetite, Emmanuel Mignot and colleagues set out to study the levels of various hormones known to regulate appetite and energy expenditure under "real life" conditions.

They took advantage of the Wisconsin Sleep Cohort Study, an ongoing longitudinal study of sleep habits and disorders in the general population. The study began in 1989, when researchers mailed state employees aged 30-60 years a survey on sleep habits, health, and demographics. Mail surveys were repeated at 5-year intervals, and some of the respondents were recruited to sleep a night in the laboratory and undergo various tests. A number of participants were also asked to keep a sleep diary for 6 days. The study has already shown connections between sleep apnea and hypertension, and between menopause and sleep-disordered breathing.

For their study, Mignot and colleagues measured sleep duration (habitual and immediately prior to blood sampling), BMI, and prebreakfast blood hormone levels in 1,024 participants. Consistent with previous studies, they found that in individuals who sleep less than 8 hours (74\% of all participants), BMI was inversely proportional to sleep duration. In addition, short sleep was associated with low leptin and high ghrelin levels (ghrelin is a hormone thought to stimulate food

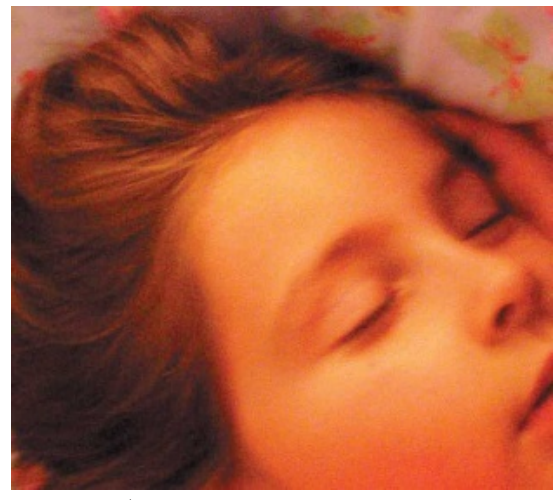

DOI: 10.1371/journal.pmed.0010068.g001

Peaceful sleep (Photo: Sharad Taheri) intake). These hormonal differences are likely to increase appetite, which could be responsible for the increased BMI in short sleepers.

These findings could explain, at least in part, why societies in which excess calories are much easier to come by than a good night's sleep are more prone to obesity. Mignot and colleagues plan to test this in intervention studies where they make people sleep more and measure the effects on body mass. "Good sleep, healthy eating habits, and regular exercise each may have important roles in fighting obesity in modern society," suggests Mignot.

Taheri S, Lin L, Austin D, Young T, Mignot E (2004) Short sleep duration is associated with reduced leptin, elevated ghrelin, and increased body mass index. DOI: 10.1371/journal.pmed.0010062 
Adverse side effects, viral resistance, and the high cost of antiretroviral therapies remain obstacles in the way of turning HIV/AIDS into a manageable chronic disease. Structured treatment interruptions (STIS) in individuals who have good viral control on therapy have been proposed as a strategy for overcoming these obstacles. The initial hope that STIs would help patients achieve greater viral control has so far not been supported by data from clinical trials, but interrupting treatment has also been proposed as a strategy to reduce the cost of longterm therapy and drug-associated toxicity.

Luis Montaner and colleagues now report results from a randomized trial of 42 participants (75\% on their second to fourth regimen, $66 \%$ on regimens containing non-nucleoside reverse-transcriptase inhibitors) who received either continuous therapy for 40 weeks or three successive treatment interruptions of two, four, and six weeks, followed by a final open-ended interruption for both groups.

The study was designed to be able to detect a difference of four weeks or greater between the two groups for the time to viral rebound during the open-ended interruption-the primary outcome. No difference between the two groups was seen (median time for the group on continuous treatment was four weeks, and for the STI group was five weeks).

Secondary outcomes included serious adverse events (disease progression, acute retroviral syndrome, therapy failure, or opportunistic infections at any point in the study), changes in CD4 count on therapy, immune reconstitution changes (CD4 recall responses and CD4 naïve/memory T cell distribution), and detection of viral mutations There were no study-related serious adverse events in either group and no increase of therapy failure in the STI arm. CD4 counts fluctuated between the start and end of each monitored treatment interruption, but levels recovered after resuppression of virus, with retention of recall responses throughout. Viral resistance was detected in both groups (in seven of 21 patients in the continuous treatment group and ten of 21 patients in the STI group), but it was more commonly detected (50\% versus 18\%) in the STI group during the open-ended final interruption, even though all subjects suppressed virus upon reinitiating the same therapy.

Possible risks and benefits of STIs remain controversial, but data from this and other published trials do not support short-term clinical benefits of treatment interruptions. However, because they do not see increased therapy failure and find preservation of immune function in the STI group, the authors conclude that, in light of the possibility of reducing costs and drug-related toxicity, additional trials of STIs are warranted.

Particularly important in the debate over the safety of STIs is whether the detection of resistant mutants should be of concern. The authors point out that all participants were able to resuppress the mutant virus when they resumed their previous drug regimens but state that it remains undetermined to what extent resistant mutations are a signal for future therapy failure. Moreover, viral replication and rebound-which eventually occurred in all participants-is seen by some researchers as inherently detrimental, and these experts argue that treatment interruptions are unsafe and their use should be discontinued.

What seems clear is that STIs have no place outside controlled clinical trials and that questions regarding long-term safety remain unanswered. At least a dozen additional trials that examine STIs are currently recruiting patients and will help answer these questions.

Papasavvas E, Kostman JR, Mounzer K, Grant RM, Gross R, et al. (2004) Randomized, controlled trial of therapy interruption in chronic HIV-1 infection. DOI: 10.1371/journal.pmed.0010064

\section{Cancer in Families}

DOI: 10.1371/journal.pmed.0010069

The Icelandic population is now a part of a unique epidemiological study, which has involved investigating the genetic heritage of many of them. The reason that this experiment can be done is because of the remarkable records that exist in Iceland. Not only is there almost complete genealogical information dating back to the 18 th century on all current $(288,000)$ and many previous Icelanders (more than 600,000 in total), but in addition the country has an almost complete cancer registry dating from 1955. A company, deCODE Genetics, was set up to mine health-care data in Iceland, and to use it to assess the effect of genetics on health. Initially, the company attracted criticism, with some questioning the ethics of providing access to healthcare data for many disease projects to a for-profit company. But the company has been supported by many Icelanders themselves, demonstrated by Icelanders donating blood samples with informed consent for research on multiple diseases, and now the project's scientific value is becoming apparent.

One such analysis is the subject of a paper by Laufey Amundadottir and colleagues in this month's PLoS Medicine that assesses how much genetic factors contribute to cancer risk across the whole Icelandic population.

The paper looked at 27 different types of cancers (all those with more than 200 cases) that had been registered between 1955 and 2002 and analysed the frequency of close and distant relatives also having that cancer, or another kind of cancer. Of the 27 cancers, 16 showed significant "familiality," and for some this risk even extended to distant

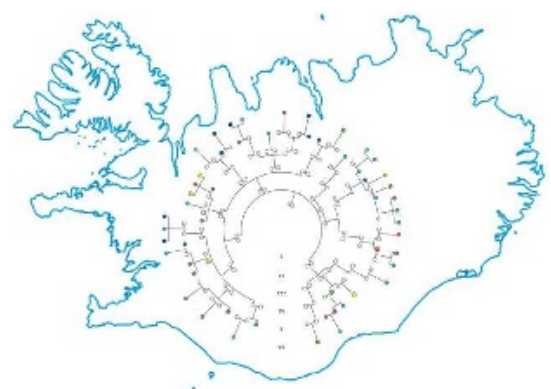

DOI: 10.1371/journal.pmed.0010069.g001

\section{Icelandic genetics and genealogy}

(that is, third- to fifth-degree) relatives. The seven cancers with the highest increased familial occurrence both in close and distant relatives were breast, prostate, stomach, lung, colon, kidney, and bladder cancers. And, interestingly, three cancers-stomach, lung, and colon 
cancer-were also seen more frequently in mates of patients, indicating a shared environmental risk factor. And for some cancers there was a familial association with other cancers, for example, relatives of individuals with stomach, colon, rectal, or endometrial cancer were more likely to have any of these cancers.

Cathryn Lewis, the academic editor for the paper comments on the study's strengths. "This level of family relationship and clinical diagnosis is rarely available from interviewing patients and family members. The size of the study (over 600,000 individuals, with 32,000 cancer cases) and the high quality of data enables the authors to detect subtle effects across distant relationships."

How robust are these data, and what do they mean for the biological understanding of cancer? As Lewis says, "Although the current study is impressive in its size and scope, even here, the sample size becomes an issue, with the most convincing results seen in the most common cancers." Certainly not all the findings are surprising; some rare cancers are already known to be associated with particular genetic defects, and syndromes that predispose to multiple cancers have been described, for example, that of Hereditary Nonpolyposis Colorectal Cancer. Other associations are more intriguing - the cluster of related cancers that include prostate, kidney, and bladder could possibly have a developmental origin, since all arise from the same part of the embryo.

So, by highlighting these subtle links, the study's particular value may become apparent: deciding future avenues of investigation in the complex interrelationships that interact to produce cancer.

Amundadottir LT, Thorvaldsson S, Gudbjartsson DF, Sulem P, Kristjansson $\mathrm{K}$, et al. (2004) Cancer as a complex phenotype: Pattern of cancer distribution within and beyond the nuclear family. DOI: 10.1371/journal.pmed.0010065 